\title{
Sierra Leone's former child soldiers: a longitudinal study of risk, protective factors, and mental health
}

\author{
Dr. Theresa S. Betancourt, Sc.D., M.A., Dr. Robert T. Brennan, Ed.D., Ed.M., Ms. Julia \\ Rubin-Smith, MSPH, Dr. Garrett M. Fitzmaurice, Sc.D., and Dr. Stephen E. Gilman, Sc.D. \\ Drs. Betancourt and Brennan and Ms. Rubin-Smith are with the François-Xavier Bagnoud Center \\ for Health \& Human Rights at the Harvard School of Public Health (HSPH); Dr. Betancourt is also \\ with the Department of Global Health \& Population at HSPH; Dr. Fitzmaurice is with the \\ Laboratory for Psychiatric Biostatistics at McLean Hospital of Harvard Medical School; Dr. Gilman \\ is with the Department of Society, Human Development \& Health and the Department of \\ Epidemiology at $\mathrm{HSPH}$
}

\begin{abstract}
Objective-To investigate the longitudinal course of internalizing and externalizing problems and adaptive/prosocial behaviors among Sierra Leonean former child soldiers and whether postconflict factors contribute to adverse or resilient mental health outcomes.
\end{abstract}

\begin{abstract}
Method-Male and female former child soldiers ( $\mathrm{N}=260$, ages 10-17 at baseline) were recruited from the roster of an NGO-run Interim Care Center in Kono District and interviewed in 2002, 2004 and 2008. The retention rate was $69 \%$. Linear growth models were used to investigate trends related to war and post-conflict experiences.
\end{abstract}

\begin{abstract}
Results-The long-term mental health of former child soldiers was associated with war experiences and post-conflict risk factors, which were partly mitigated by post-conflict protective factors. Increases in externalizing behavior were associated with killing/injuring others during the war and post-conflict stigma while increased community acceptance was associated with decreases in externalizing problems $(\mathrm{B}=-1.09)$. High baseline levels of internalizing problems were associated with surviving rape while increases were associated with younger involvement in armed groups and social and economic hardships. Improvements in internalizing problems were associated with higher levels of community acceptance and increases in community acceptance $(\mathrm{B}=-0.86)$. Decreases in adaptive/prosocial behaviors were associated with killing/injuring others during the war and post-conflict stigma, but partially mitigated by social support, being in school and increased community acceptance $(\mathrm{B}=1.93)$.
\end{abstract}

Conclusions-Psychosocial interventions for former child soldiers may be more effective if they account for post-conflict factors in addition to war exposures. Youth with accumulated risk factors, lack of protective factors, and persistent distress should be identified; sustainable services

\footnotetext{
(C) 2011 American Academy of Child \& Adolescent Psychiatry. Published by Elsevier Inc. All rights reserved

Correspondence to Dr. Theresa S. Betancourt, Research Program on Children and Global Adversity/François-Xavier Bagnoud Center for Health \& Human Rights, Harvard School of Public Health, 651 Huntington Avenue, 7th floor, Boston, MA 02115 USA, Tel: 617 432-5003; Fax: 617 432-4310; Theresa_Betancourt@harvard.edu.

Disclosure: Drs. Betancourt, Brennan, Fitzmaurice, Gilman, and Ms. Rubin-Smith report no biomedical financial interests or potential conflicts of interest.

Publisher's Disclaimer: This is a PDF file of an unedited manuscript that has been accepted for publication. As a service to our customers we are providing this early version of the manuscript. The manuscript will undergo copyediting, typesetting, and review of the resulting proof before it is published in its final citable form. Please note that during the production process errors may be discovered which could affect the content, and all legal disclaimers that apply to the journal pertain.
} 
to promote community acceptance, reduce stigma, and expand social supports and educational access are recommended.

\section{Keywords}

Child soldiers; internalizing problems; externalizing problems; prosocial behaviors; longitudinal study

\section{INTRODUCTION}

Globally, an estimated 300,000 children under the age of 18 are involved with armed forces and armed groups. ${ }^{1,2}$ Despite the documentation of risks facing child soldiers due to warrelated violence, ${ }^{3}$ little is known about what influences long-term mental health trajectories and processes of social reintegration. Recent studies on former child soldiers from northern Uganda, ${ }^{4}$ the Democratic Republic of the Congo, ${ }^{5}$ and $\mathrm{Nepal}^{6}$ have provided insight into the impact of war experience on reintegration and psychosocial adjustment. Research documents that witnessing, experiencing and perpetrating violence, younger age of involvement, and longer engagement with an armed group all have negative consequences for the mental health and social reintegration of young people. ${ }^{5-7}$ Although loss and displacement are common adversities confronted by all conflict-affected children, ${ }^{8}$ child soldiers face additional risks such as exceptionally prolonged and intense exposure to violence. ${ }^{9}$ This exposure can include being forced to kill or harm others ${ }^{1,10}$ and repeated personal victimization, including sexual violence. ${ }^{10}$

Prior studies have documented high rates of mental health problems, such as PTSD and depression, among former child soldiers. ${ }^{4}{ }^{11}$ However, wartime exposures alone do not account for the elevated burden of mental health problems in these young people $e^{6,7,12,13}$ raising the question of how post-conflict factors may contribute to varying degrees of vulnerability to adverse outcomes. One longitudinal study documented that post-conflict experiences such as family support and economic opportunity played a role in the mental health of 39 Mozambican males reinterviewed 16 years after reintegration. ${ }^{12}$ More recently, in northern Uganda, research by Blattman and Annan ${ }^{7}$ emphasized that widespread educational and economic deprivation contribute significantly to adverse outcomes in former child soldiers. In Nepal, Kohrt et al. concluded that post-conflict factors such as stigma might contribute to adverse mental health outcomes. Former child soldiers in his sample showed significantly higher symptoms of depression and PTSD compared to matched controls even after adjusting for exposure to traumatic events. ${ }^{6}$ Similarly, Betancourt and colleagues in Sierra Leone observed that post-conflict experiences of discrimination may significantly explain the relationship between past involvement in wounding/killing others and subsequent increases in hostility. Stigma also mediated the relationship between surviving rape and increases in depression over a two year follow-up period. $^{13}$

The present study builds on prior research by investigating the role of post-conflict risk and protective factors in the relationship between war experiences and mental health in a cohort of male and female former child soldiers followed prospectively over three time points. We hypothesized that ongoing risk factors such as stigma and daily hardships would contribute to poor mental health outcomes while protective factors such as being in school, working, or experiencing social support and community acceptance would contribute to improved mental health outcomes. 


\section{METHOD}

\section{Study Cohort and Procedures}

This prospective longitudinal study was conducted in collaboration with the International Rescue Committee (IRC) and the Post-conflict Reintegration Initiative for Development and Empowerment (PRIDE). Survey interviews were conducted at three time points: T1 (2002), T2 (2004) and T3 (2008). Subjects were children who had been involved with the Revolutionary United Front (RUF) and who had then been referred to the IRC's Disarmament, Demobilization, and Reintegration (DDR) program in Sierra Leone's Kono District. ${ }^{14}$ The IRC's Interim Care Center (ICC) served five districts of Sierra Leone; the sample was obtained by pooling IRC registries to create a master list of all youth $(\mathrm{N}=309)$ assisted by the ICC from June 2001 to February 2002, the most active period of demobilization. Youth who were between the ages of 10 and 17 in 2002 and who had contact information available were approached and invited to participate in the baseline assessment $(\mathrm{N}=260)$. At baseline, no youth and no caregivers refused consent/assent. At $\mathrm{T} 2$, $\mathrm{N}=147$ (56.5\%) of the sample had been reinterviewed when data collection was terminated due to the death of our collaborating NGO's country director. At T3, we were able to recontact $68.8 \%$ of the original sample, including many we did not interview at T2. At T1 $55.3 \%(\mathrm{~N}=256)$ percent of youth lived with at least one biological parent, while this percentage was $53.7 \%(\mathrm{~N}=147)$ at $\mathrm{T} 2$ and $34.1 \%(\mathrm{~N}=179)$ at $\mathrm{T} 3$. At all times of assessment participants were in a home situation with an identified parent or guardian who was legally able to give consent for the child to participate. No children lived in institutionalized settings or situations where additional consent from government guardians was needed. $47.3 \%$ of the original sample were assessed at all three waves while $30.8 \%$ of the sample was assessed at two waves (either at $\mathrm{T} 1$ and $\mathrm{T} 2$ or at $\mathrm{T} 1$ and $\mathrm{T} 3$ ) and $21.9 \%$ of the sample was assessed only at T1.

Trained Sierra Leonean research assistants conducted private face-to-face interviews, first with subjects and then with index caregivers. Because of low literacy in the population, all consents/assents and study protocols were administered verbally in Krio, the most widelyspoken language in Sierra Leone. Interviews lasted 1-3 hours. Survey protocols were approved by internal review at the IRC (for T1) and IRB committees at the Boston University School of Medicine/Boston Medical Center (for T2) and the Harvard School of Public Health (for T3). Social workers traveled with the research team at all waves to respond to serious emotional or physical health needs. During T1 and T2 youth who showed signs of immediate risk of harm received visits from IRC social workers. At T3, 5\% percent of those interviewed were determined to be at immediate risk of harm (mainly due to suicidal ideation) and were referred to mental health services via the Community Assistance for Psychosocial Support (CAPS) program in Kono.

\section{Study Instruments}

In diverse cultural settings such as post-conflict Sierra Leone, the assessment of mental health outcomes remains a persistent challenge, as constructs must be identified, framed and measured in culturally meaningful and valid ways. ${ }^{15-17}$ We used a mix of standard measures and locally-derived measures, developed for use in Sierra Leone. ${ }^{18}$ All measures were selected and adapted in close consultation with local staff and community members. Focus groups of youth and adults were used to develop additional questionnaire items and to determine the face validity and cultural relevance of standard measures. Similar combined methods for cross-cultural instrument development have been used in recent studies of former child soldiers. ${ }^{6}$ All measures were forward- and back-translated to ensure accuracy following a standard protocol. ${ }^{19}$ Given that several study measures were used across a wide developmental range, we examined a number of factors related to the psychometric 
properties of the measures at each time point. We observed good internal consistency and expectable indicators of concurrent and predictive validity at each time point (i.e. strong correlation between conceptually related scales such as the locally-derived scales and scales validated for assessing depression and anxiety in adults). Variables expected to be correlated for conceptual reasons also demonstrated expected correlations (for example, at T3 internalizing and externalizing symptoms were significantly and inversely associated with community acceptance $(r=-0.21$ and $r=-0.22, p \leq 0.01)$ and positively associated with adaptive behaviors $(\mathrm{r}=.44, p \leq 0.001)$ ). We also examined the correlation between the three outcomes of interest. Across all waves, the correlation between internalizing and externalizing ranged from $0.43-0.67$. The correlation between adaptive/prosocial behavior and internalizing ranged from -0.24 to 0.03 and between adaptive prosocial behavior and externalizing ranged from -0.02 to 0.14 .

Mental Health Assessment-Mental health was assessed across all three waves using the Oxford Measure of Psychosocial Adjustment developed and validated for use among former child soldiers in Sierra Leone and northern Uganda. ${ }^{18,}{ }^{20}$ Six items were excluded from the original 52-item scale because they did not contribute to the internal consistency of subscales. The reduced version of the instrument was arranged into subscales for externalizing problems (hostility, 12 items, range 0-48, T1 to T3 average Cronbach's $\alpha=0.81$ ), internalizing problems (anxiety and depression, 16 items, range $0-64$, T1 to T3 average Cronbach's $\alpha=0.79$ ), and a subscale of adaptive/prosocial behaviors (confidence and prosocial behaviors, 18 items, range $0-72$, T1 to T3 average Cronbach's $\alpha=0.84$ ). Such dimensional approaches assess emotional and behavioral problems along a continuum, rather than solely on diagnostic categories. They are considered to be more sensitive to child development as well as to cross cultural differences and, most important to our study, to change over time. ${ }^{16}$ Within a dimensional approach, internalizing problems refer to inwarddirected experiences of distress commonly related to symptoms of depression or anxiety. Externalizing problems refer to outward-directed behaviors such as hostility and aggression. A sum of the total problem score on the Oxford Measure of Psychosocial Adjustment showed significant correlations with standard measures of psychological problems including the 9-item version of the Child Posttraumatic Stress Disorder Reaction Index (PTSD-RI) ${ }^{21}$ (T1 r=0.47, p<0.001; T2 r=0.68, p<0.0001) and the 25-item Hopkins Symptom Checklist/ $S F-25$ (HSCL-25) 22 (T1 r=0.51, p <0.001; T2 r=0.68, p<0.0001). These standard measures have not been validated for use in Sierra Leone, but comparison indicates correlation in the expected direction between the core outcome measure used in this study and standard scales that have been employed to assess the mental health of war-affected youth in other international settings. 4,23

An adapted version of the Inventory of Socially Supportive Behaviors, consisting of twentyone items assessing perceived emotional, instrumental and informational support from others (range 0-84, T3 Cronbach's $\alpha=0.87$ ), was added at T3. ${ }^{24}$ The scale of Community Acceptance, administered at all three waves, consisted of six items developed from locallycollected qualitative data and assessed the manner in which subjects perceived community acceptance. These items were not phrased to refer specifically to the experience of being a child soldier (range $0-12$, T1 to T3 average Cronbach's $\alpha=0.89$ ).

At T2 (and at T3 for subjects not interviewed at T2), items from the Child War Trauma Questionnaire ${ }^{25}$ were selected to assess individual war experiences. Three types of war experiences were examined in light of theory and existing research on mental health in former child soldiers: 1) witnessing general war violence (e.g., massacres or raids on villages); 2) being a victim of rape; and 3) injuring or killing others. ${ }^{11}$ In particular, stressors such as surviving rape and injuring/killing others may be considered particularly "toxic" and may greatly threaten the development and mental health of formerly abducted children. ${ }^{9}$ The 
survey instrument also included two self-reported classifications: age of abduction and duration of time with the fighting forces.

The Post-War Adversities Index ${ }^{26}$ was adapted to assess daily hardships facing youth in post-conflict Sierra Leone. Sixteen items (range 0-16) were used to determine housing and economic insecurity (10 items), as well as to assess interpersonal adversities, such as living with people who fight or use drugs (6 items). Demographic and household information such as gender, age, and socioeconomic status (SES) (an 8-item measure pertaining to family resources and property), was also collected via youth self-report. At each wave of assessment, subjects were asked whether they were attending school, and at T3 they were also asked if they were employed. In order to allow comparisons between predictors with dissimilar scales of measurement, the following variables were standardized (mean=0; standard deviation=1): SES, number of violent events witnessed, stigma score, social support and community acceptance; all other variables, including outcomes and predictors, are in their original metrics.

Stigma and perceived discrimination at $\mathrm{T} 2$ and $\mathrm{T} 3$ were assessed with the Everyday Discrimination Scale ${ }^{27}$ comprising 9 items that capture negative community interactions such as differential treatment, threats, or abuse. Responses were scored on a 0-2 scale, ranging from "never=0," "sometimes $=1$ " to "always $=2$. ." For each item endorsed as "sometimes" or "always," interviewers probed the reason for the perceived discrimination. Though eight potential reasons for discrimination were given (status as a child soldier, religion, gender, age, disability, poverty, level of education, and tribe) only stigma due to being a former child soldier was used in the present analysis (range $0-18$, T2 to T3 average Cronbach's $\alpha=0.89$ ). Although we recognize that numerous reasons for discrimination are of potential importance, we chose to focus on discrimination due to being a child soldier (which was the most frequently endorsed) for the purposes of parsimony and to illuminate phenomena specific to child soldiers in the present analysis.

\section{Statistical Analyses}

Multilevel linear growth modeling was used to relate study participants' mental health outcomes over time to war experiences, as well as to post-conflict risk and protective factors. In these models, trajectories of mental health outcomes were represented by two parameters: an intercept, which quantifies a young person's baseline level of mental health, and slope, which quantifies changes in mental health over time. ${ }^{28}$ Coefficients for predictors of the slope indicate the relationship between time-invariant predictors and changes in mental health over time, wherein a positive coefficient indicates that larger predictor values are associated with an increase in the outcome over time. For consistency in interpreting coefficients across models, all predictors were centered on their grand means for both the intercept and the time slopes. Therefore, regression coefficients are interpreted in terms of deviations from the sample grand mean.

Analyses also included two time-varying predictors - school participation and community acceptance - that were measured at all three time points. An increase in these variables could relate directly to increases or decreases in outcomes over time. Each of these timevarying predictors has a time-invariant counterpart (the within-person mean). The timeinvariant component for community acceptance is the average value of community acceptance across the three time points; for school attendance, it is the total number of time points at which school attendance was reported. In each case, the time-varying component is then centered on the within-person mean value to represent change only.

Each mental health outcome was fit with three models: the first included war experiences; the second added post-war adversities including daily hardships and stigma due to being a 
former child soldier; and the third added post-war protective factors including community acceptance, social support, school attendance, and employment. Models also controlled for age, sex, and SES. In the second and third models, a deviance test measured whether the added variables improved model fit over the previous model. The war experiences model was compared to a model containing only demographic covariates. All growth models were estimated with HLM 6.0 Software. ${ }^{29}$

Missing data were addressed by multiple imputation (MI) at each time point only for those subjects interviewed at that time point. This approach reduces bias to the extent that values on the observed variables are informative about the likelihood an item was missing; increases precision relative to a complete case analysis with a smaller sample size; and accounts for the sampling variability across imputations. ${ }^{30}$ Nine imputed datasets were generated using the method of chained equations as implemented in IVEware ${ }^{31}$ and analyzed using the multiple imputation feature of the HLM software. ${ }^{28}$ Further, multilevel growth modeling allows inclusion of incomplete cases so cases with completely missing time points are included in the models, but only with data on the time points that are observed for a given subject. ${ }^{28,29}$

\section{RESULTS}

\section{Characteristics of Participants}

Table 1 displays characteristics of the sample of former child soldiers $(\mathrm{N}=260)$. The sample was predominantly male $(88.8 \%, \mathrm{n}=231)$, and nearly all participants were either Christian $(52.3 \%, \mathrm{n}=136)$ or Muslim $(47.3 \%, \mathrm{n}=123)$. Virtually all youth in the sample reported joining the RUF by force/abduction $(97.7 \%, \mathrm{n}=254)$. Youth joined the rebels at a mean age of 10.3 years and remained an average of 4.1 years. Participants had witnessed an average of 6.2 war-related violent events. Forty-five percent of girls and 5\% of boys reported being raped during their time with the rebels. More than a quarter of the sample $(26.9 \%, \mathrm{n}=70)$ reported having killed or injured others during the war. Comparisons between subjects retained and those lost to follow up (results not shown) revealed no significant differences on variables of interest, with the exception of school attendance whereby a higher proportion of completers versus non-completers (69\% vs. $47 \%)$ were in school at baseline $(\mathrm{p}=0.003)$.

\section{Retention}

Of eligible subjects approached for interviews at T2, one caregiver declined consent for his/ her child to participate, one subject had died and 19 subjects had moved to distant locations that precluded follow-up. Midway through T2 data collection, the death of IRC's country director suspended program activities and halted the study, preventing further participant follow-up. As a result, a total of 113 participants (43.5\% of the original sample) were not recontacted at T2. When the third wave of assessment was conducted in 2008 (T3), attempts were made to re-interview all 260 from the original sample. Overall, 81 participants (31.2\% of the original sample) were lost to follow up at T3; 68 participants could not be found due to insufficient contact information, 4 had moved to another country, and 9 were deceased.

\section{Growth models of children's mental health problems and adaptive/prosocial behaviors}

The results of linear growth models for each mental health outcome over time are shown in Table 2. Deviance tests conducted at each stage - war experiences, post-conflict hardships, and post-conflict protective factors - indicated that each subsequent model fit better than the previous one for all three outcomes.

Externalizing Problems-Youth who experienced stigma due to being a child soldier had higher baseline levels of externalizing problems $(b=0.89, \mathrm{p}=0.016)$ in models 
considering only war experiences (adjusted for covariates). However, this association was no longer statistically significant $(b=0.73, \mathrm{p}=0.058)$ upon consideration of post-conflict protective factors. Looking at change over time, child soldiers who had injured or killed others demonstrated significant increases in externalizing problems $(b=1.16, \mathrm{p}=0.031)$. However, after adjusting for post-conflict risk factors such as stigma and daily hardships, this effect did not retain statistical significance, suggesting that the association between wartime experience on externalizing is additionally explained by post-conflict factors. Of the protective factors examined, increased community acceptance was associated with decreased externalizing problems $(b=-1.09, \mathrm{p}<0.001)$, such that a one standard deviation (SD) increase in the community acceptance score was associated with a 0.10 SD decrease in the externalizing problems score.

Internalizing Problems-In models examining internalizing problems (anxiety/ depression), being raped, longer periods of time with an armed group and being subjected to stigma were associated with higher baseline levels of internalizing problems. After adjusting for all hardship and protective factors, among time-invariant predictors, only being raped remained significant $(b=4.34, \mathrm{p}=0.039)$. Higher average levels of community acceptance were significantly and inversely associated with lower baseline levels of internalizing problems $(b=1.21, \mathrm{p}=0.003)$.

Looking at change over time, youth who had become involved with fighting forces at a younger age showed increases in internalizing problems $(b=-0.35$ for each year of age, $\mathrm{p}=0.02$ ). Thus, being 3 years younger at the time of abduction translated into an increase between time points of $0.10 \mathrm{SD}$ in internalizing problems. Higher levels of daily hardships were also associated with increased internalizing problems such that a one unit greater postconflict hardships score was associated with a 1.38 unit increase on the internalizing problems scale over time $(\mathrm{p}<.001)$. Looking to protective factors in the post-conflict environment, community acceptance again demonstrated a protective relationship such that each additional SD increase in community acceptance over time was associated with a 0.13 SD decrease in internalizing problems.

Adaptive/Prosocial Attitudes and Behaviors-For positive developmental outcomes, we observed that retention in school and higher levels of community acceptance were associated with higher baseline levels of adaptive/prosocial behavior $(b=1.50, \mathrm{p}<0.001$ and $b=1.69, \mathrm{p}<0.001$ ) (see predictors of "Baseline" slope in Table 2). However, over time, child soldiers who had injured or killed others had significant decreases in prosocial/adaptive behaviors $(b=-2.60, \mathrm{p}<0.001)$ adjusting for covariates. After adding post-conflict risk factors such as stigma and daily hardships to the model, the association between injuring/ killing others and adaptive/prosocial behaviors was reduced, but remained significant $(b=$ $-1.88, \mathrm{p}=0.02$ ). Thus, post-conflict stigma due to being a child soldier was associated with a decline in adaptive/prosocial behaviors $(b=-0.91 \mathrm{p}=0.01)$ over time. However, the magnitude of the association between stigma and declines in adaptive/prosocial behavior was no longer statistically significant upon adding post-conflict protective factors to the model $(b=-0.61, \mathrm{p}=0.054)$. In addition, after adjusting for post-conflict protective factors, the reduction in prosocial behaviors associated with having killed/injured others in war was further reduced to about one half the original effect $(b=-1.29, \mathrm{p}=0.068)$. Of the protective factors examined, social support $(b=0.93, \mathrm{p}=0.006)$ and increasing community acceptance $(b=1.93, \mathrm{p}<0.001)$ were associated with increased prosocial/adaptive behaviors over time.

\section{DISCUSSION}

Former child soldiers' acute war experiences have long-term consequences, but the nature and extent of these consequences are influenced by post-conflict risk and protective factors. 
In our sample, injuring/killing others was associated with increasing externalizing problems and decreasing levels of adaptive/prosocial behaviors while being raped predicted higher baseline levels of internalizing problems (anxiety/depression). However, most of the associations between these war experiences and longitudinal outcomes were not statistically significant in models that accounted for post-conflict factors. The relationships between acute war trauma, internalizing and externalizing problems, and positive psychosocial adjustment were therefore significantly shaped by post-conflict experiences.

Our results also suggest that different forms of trauma have differing degrees and types of long-term mental health impact. General witnessing of war violence, for example, was not associated with changes in mental health, while being raped was predictive of higher levels of internalizing problems but not of increased externalizing problems or decreased prosocial behavior. Our findings - particularly the associations we observed between perpetration of violence, increases in externalizing problems, and decreases in adaptive/prosocial behaviors - are consistent with relationships observed in other studies of former child soldiers ${ }^{3,5}$ and adult war veterans. ${ }^{32}$

Our findings point to a number of important post-conflict factors that influence long-term wellbeing and that may represent appropriate targets for intervention. Youth who remained in school and those who experienced higher levels of community acceptance had higher baseline levels of adaptive/prosocial behaviors adjusting for all other factors. In addition, decreases in adaptive/prosocial behaviors were associated with higher levels of stigma, while increases were associated with social support and school attendance. Most promisingly, in the presence of increasing community acceptance, youth demonstrated significant improvements in all outcomes investigated.

Taken together, these findings highlight the potential of post-conflict intervention programs that address community dynamics such as acceptance and stigma and that bolster strengths at the individual level through social support. In Sierra Leone, thoughtful attention was given to promoting community acceptance of former child soldiers immediately following the war. ${ }^{14}$ Community sensitization campaigns assisted the initial phases of reintegration but did not continue over the long-term. ${ }^{33}$ The nationwide sensitization efforts were particularly strong before the creation of the Sierra Leone Truth \& Reconciliation Commission, which was meant to coordinate mass campaigns. Given the long-term positive effects of improved community acceptance on former child soldiers' mental health, interventions aimed at bolstering and maintaining this protective process may strengthen and sustain the impact of initial efforts.

Our findings underscore aspects of community-based intervention features - including efforts to reduce stigma, increase community acceptance and increase school attendance that are crucial to the mental well-being of war-affected youth. However, despite how our findings support broad, community-based approaches, we do not disregard the possible beneficial effects of targeting interventions at individual child soldiers. Without specific comparison groups (i.e. a sample of non-war-exposed children with behavioral problems), there is not sufficient evidence to rule out war-related factors as ultimately etiological and influential in subsequent post-conflict adjustment.

Study limitations include moderate retention rates given the challenging field conditions and early termination of the $\mathrm{T} 2$ data collection, use of self-reports and a lack of information on pre-war levels of mental health problems in Sierra Leone, which limit our ability to interpret the magnitude of the mental health problems observed. Furthermore, although our measure of mental health outcomes was developed and validated for use in Sierra Leone, clinical cut points are unavailable for this context. Additionally, we cannot make strong claims about 
causality in these data since war-related experiences were reported retrospectively. Our findings are also limited in their general application to the country as a whole, as our sample comprises youth who originated from 5 out of 14 districts in Sierra Leone (Kono, Bo, Kenema, Pujehun, and Moyamba) demobilized over a specified time period; this design was necessitated in part by the significant ethical and logistical difficulties involved in obtaining a country-level representative sample of former child soldiers. However, we have no reason to believe that the children we interviewed are very different from either the children in their own districts or other districts that were served via the DDR process. Finally, without a comparison group of self-reintegrated youth or youth never associated with armed groups, we cannot broadly generalize our findings to comment on other groups of war-affected youth.

Study strengths include the use of dimensional assessments capturing a broad range of mental health indicators in a culturally sensitive manner as opposed to a narrow focus on trauma-related disorders. Moreover, by including multiple types of outcome measures, including positive/adaptive outcomes, we were able to identify different patterns of sensitivity. For example, all outcomes were associated with community acceptance, but rape was more associated with internalizing problems while killing/injuring others was more associated with externalizing problems and deficits in prosocial/adaptive behaviors. While stigma influenced both internalizing and externalizing problems, internalizing problems were additionally influenced by daily hardships and age of involvement with armed groups. Adaptive/prosocial behavior, meanwhile, was most sensitive to school access and social support.

Overall, this prospective study contributes to the literature on the processes that influence mental health in former child soldiers over time. By examining post-conflict variables, we identified broader processes that work to shape the long-term psychosocial adjustment and well being of children associated with armed forces and armed groups; in short, we found that mental health in this group is influenced by much more than past war experiences. Although our general finding may apply broadly, the specific processes that influence risk and resilience are likely to be shaped by culture and context. ${ }^{34}$ Although stigma and discrimination may be seen as universally detrimental human experiences, the social meaning of being a former child soldier may differ, for example, in a context where youth involvement in conflict is focused on joining together to fight a common enemy compared to situations where forced abduction plays a significant role. Similarly, although the experience of being raped may be considered universally harmful, the implications of this experience certainly interact with gender and culture to influence adverse outcomes. ${ }^{10} \mathrm{In}$ Sierra Leone, other research has observed that, although both boys and girls reported rape, stigma against girls more commonly included insinuations of sexual impropriety that are particularly damaging to the social prospects of females. ${ }^{13,}{ }^{35}$ In this manner, the nature of both war experiences and post-conflict experiences must be examined carefully across settings.

Based on our findings, we suggest that post-conflict adversities and resources must be given closer attention in both research and service delivery. Such understanding of the impact of multiple war-related and post-conflict factors is important for identifying appropriate intervention targets. The results of this study emphasize the importance of investing in longer-term (i.e., beyond the immediate post-conflict period) approaches to monitoring and supporting the wellbeing of war-affected youth. 


\section{Acknowledgments}

This study was funded by the United States Institute of Peace, USAID/DCOF, Grant \#1K01MH077246-01A2 from the National Institute of Mental Health, the International Rescue Committee and the François-Xavier Bagnoud Center for Health and Human Rights.

The authors would like to thank Sidney Atwood, Kathleen McGaffigan and Laura Khan for their assistance in data management and analysis. Mr. Atwood is with the Division of Global Health Equity at Brigham \& Women's Hospital; Ms. McGaffigan is with the Harvard School of Public Health; Ms. Khan is a student at HSPH.

\section{References}

1. Coalition to Stop the Use of Child Soldiers. Child soldiers: Global report 2008. London: 2008.

2. Lancet. The hidden health trauma of child soldiers. Lancet. 2004; 363:831. [PubMed: 15031020]

3. Betancourt TS, Borisova II, Brennan RB, et al. Sierra Leone's former child soldiers: A follow-up study of psychosocial adjustment and community reintegration. Child development. In press.

4. Derluyn I, Broekaert E, Schuyten G, De Temmerman E. Post-traumatic stress in former Ugandan child soldiers. Lancet. Mar 13; 2004 363(9412):861-863. [PubMed: 15031032]

5. Bayer CP, Klasen F, Adam H. Association of trauma and PTSD symptoms with openness to reconciliation and feelings of revenge among former Ugandan and Congolese child soldiers. JAMA. Aug 1; 2007 298(5):555-559. [PubMed: 17666676]

6. Kohrt BA, Jordans MJD, Tol WA, et al. Comparison of mental health between former child soldiers and children never conscripted by armed groups in Nepal. JAMA. August 13; 2008 300(6):691702. [PubMed: 18698067]

7. Blattman C, Annan J. The consequences of child soldiering. Review of Economics and Statistics. in press.

8. Lustig SL, Kia-Keating M, Knight WG, et al. Review of child and adolescent refugee mental health. Journal of the American Academy of Child and Adolescent Psychiatry. Jan; 2004 43(1):24-36. [PubMed: 14691358]

9. National Scientific Council on the Developing Child. Excessive Stress Disrupts the Architecture of the Developing Brain: Working Paper \#3. Cambridge, MA: The Center on the Developing Child at Harvard University; 2005.

10. Johnson K, Asher J, Rosborough S, et al. Association of combatant status and sexual violence with health and mental health outcomes in postconflict Liberia. JAMA. Aug; 2008 300(6):676-690. [PubMed: 18698066]

11. Betancourt, TS.; Borisova, I.; Rubin-Smith, JE.; Gingerich, T.; Williams, T.; Agnew-Blais, J. Psychosocial adjustment and social reintegration of children associated with armed forces and armed groups: The state of the field and future directions. Austin, TX: Psychology Beyond Borders; 2008.

12. Boothby N, Crawford J, Halperin J. Mozambique child soldier life outcome study: Lessons learned in rehabilitation and reintegration efforts. Global Public Health. 2006; 1(1):87-107. [PubMed: 19153896]

13. Betancourt TS, Agnew-Blais J, Gilman SE, Williams DR, Ellis BH. Past horrors, present struggles: the role of stigma in the association between war experiences and psychosocial adjustment among former child soldiers in Sierra Leone. Soc Sci Med. Jan; 2010 70(1):17-26. [PubMed: 19875215]

14. Williamson J. The disarmament, demobilization and reintegration of child soldiers: Social and psychological transformation in Sierra Leone. Intervention: The International Journal of Mental Health, Psychosocial Work and Counselling in Areas of Armed Conflict. 2006; 4(3):185-205.

15. Canino G, Alegria M. Psychiatric diagnosis - is it universal or relative to culture? Journal of child psychology and psychiatry, and allied disciplines. 2008; 49(3):237-250.

16. Achenbach, TM.; Rescorla, LA. Multicultural Understanding of Child and Adolescent Psychopathology: Implications for Mental Health Assessment. New York: The Guilford Press; 2006. 
17. Betancourt TS, Speelman L, Onyango G, Bolton P. A qualitative study of psychosocial problems of war-affected youth in northern Uganda. Journal of Transcultural Psychiatry. June; 2009 46(2): 238-256.

18. MacMullin C, Loughry M. Investigating psychosocial adjustment of former child soldiers in Sierra Leone and Uganda. Journal of Refugee Studies. 2004; 17(4):460-472.

19. Van Ommeren M, Sharma B, Thapa S, et al. Preparing instruments for transcultural research: Use of the translation monitoring form with Nepali-speaking Bhutanese refugees. Transcultural Psychiatry. 1999; 36(3):285.

20. MacMullin, C.; Loughry, M. Proposal to Assess Psychosocial Adjustment of Former Child Soldiers in Sierra Leone; Phase 1 Final Report: Construction of Research Instrument. New York, NY: International Rescue Committee; 2000.

21. Pynoos, RS.; Rodriguez, N.; Steinberg, AS.; Stauber, M.; Frederick, C. UCLA PTSD Index for DSM-IV child version. Los Angeles, CA: UCLA Psychiatry Trauma Service; 1998.

22. Derogatis LR, Lipman RS, Rickels K, Uhlenhuth EH, Covi L. The Hopkins Symptom Checklist (HSCL): a self-report symptom inventory. Behavioral Science. Jan; 1974 19(1):1-15. [PubMed: 4808738]

23. Layne, CM.; Olsen, JA.; Baker, A.; Legerski, JP.; Isakson, B.; Pašaliuć, A. Unpacking trauma exposure risk factors and differential pathways of influence: Predicting post-war mental distress in Bosnian adolescents. In press

24. Barrera M, Ainlay SL. The structure of social support: A conceptual and empirical analysis. Journal of Community Psychology. April.1983:11.

25. Macksoud MS. Assessing war trauma in children: A case study of Lebanese children. Journal of Refugee Studies. 1992; 5(1):1-15.

26. Layne, CM.; Stuvland, R.; Saltzman, W.; Djapo, N.; Pynoos, RS. Unpublished instrument. 1999. Adolescent Post War Adversities Scale.

27. Williams DR, Yu Y, Jackson JS, Anderson NB. Racial differences in physical \& mental health: Socioeconomic status, stress, and discrimination. Journal of Health Psychology. 1997; 2(3):335351.

28. Singer, JD.; Willett, JB. Applied Longitudinal Data Analysis: Modeling Change and Event Occurrence. New York: Oxford University Press; 2004.

29. Raudenbush, SW.; Bryk, AS.; Cheong, YF.; Congdon, RT. HLM 6: Hierarchical linear and nonlinear modeling. 2. Chicago, IL: Scientific Software International; 2005.

30. Rubin, DB. Multiple imputation for nonresponse in surveys. New York: Wiley \& Sons; 1987.

31. Raghunathan TE, Lepkowski JM, Van Hoewyk J, Solenberger P. A multivariate technique for multiply imputing missing values using a sequence of regression models. Survey Methodology. 2001; 27(1):85-95.

32. Hofmann SG, Litz BT, Weathers FW. Social anxiety, depression, and PTSD in Vietnam veterans. Journal of Anxiety Disorders. 2003; 17(5):573. [PubMed: 12941367]

33. Zaeh, S.; Betancourt, T. Mental Health and Psychosocial Services for Youth in Post-Conflict Sierra Leone [case study]. In progress

34. Betancourt TS, Khan KT. The mental health of children affected by armed conflict: Protective processes and pathways to resilience. International Review of Psychiatry. Jun; 2008 20(3):317328. [PubMed: 18569183]

35. Denov M, Gervais C. Negotiating (In)Security: Agency, resistance, and resourcefulness among girls formerly associated with Sierra Leone's Revolutionary United Front. Signs: Journal of Women in Culture \& Society. Summer;2007 32(4):885-910. 


\section{Table 1}

Characteristics of the sample (former child soldiers in Sierra Leone) and distributions of main variables at each assessment reported as Mean (SD) or frequency (\%).

\begin{tabular}{|l|c|c|c|c|}
\hline & Range of Scale & T1 N=260 & T2 N=147 & T3 N=179 \\
\hline Age & & $15.13(2.22)$ & $17.41(2.38)$ & $21.75(3.16)$ \\
\hline Outcomes & & & & \\
\hline Externalizing problems & $0-48$ & $19.32(5.18)$ & $20.24(6.30)$ & $18.52(4.44)$ \\
\hline Internalizing problems & $0-64$ & $34.54(7.64)$ & $35.44(7.63)$ & $34.83(6.45)$ \\
\hline Adaptive/prosocial behaviors & $0-72$ & $58.27(7.30)$ & $59.29(7.46)$ & $57.42(7.21)$ \\
\hline Post-Conflict Hardships & & & & \\
\hline Stigma due to being a child soldier & $0-18$ & $\ldots$ & $2.50(3.63)$ & $1.61(3.09)$ \\
\hline Post-conflict hardships & $0-16$ & $\ldots$ & $\ldots$ & $5.03(3.54)$ \\
\hline Post-Conflict Protective Factors & & & & \\
\hline Social support & $0-84$ & $\ldots$ & $\ldots$ & $41.32(13.45)$ \\
\hline Community acceptance & $0-12$ & $10.50(2.55)$ & $10.38(2.41)$ & $9.85(2.71)$ \\
\hline In school at time of assessment & $\ldots$ & $165(63.5 \%)$ & $111(75.5 \%)$ & $86(48.9 \%)$ \\
\hline Working & $\ldots$ & $\ldots$ & $\ldots$ & $43(24.0 \%)$ \\
\hline
\end{tabular}

Note: $\mathrm{N}=$ number of participants with complete data available at each time point that were included in analyses. $\mathrm{T} 1=2002, \mathrm{~T} 2=2004, \mathrm{~T} 3=2008$. 




J Am Acad Child Adolesc Psychiatry. Author manuscript; available in PMC 2011 August 17. 




Page 14

J Am Acad Child Adolesc Psychiatry. Author manuscript; available in PMC 2011 August 17. 